\title{
Protective effect of sevoflurane on myocardial ischemia-reperfusion injury in rat hearts and its impact on HIF-1 $\alpha$ and caspase-3 expression
}

\author{
TAO ZHOU, SHANLIANG GUO, SHAOLIN WANG, QIONG LI and MINGSHENG ZHANG
}

Department of Anesthesiology, Jiangxi Provincial People's Hospital, Nanchang, Jiangxi 330006, P.R. China

Received May 15, 2017; Accepted August 18, 2017

DOI: $10.3892 / \mathrm{etm} .2017 .5078$

\begin{abstract}
This study was designed to investigate possible protective effects of sevoflurane on myocardial ischemiareperfusion injury (MIRI) and its impact on expression of HIF-1 $\alpha$ and caspase-3 in rats, so as to provide new insights for the treatment of MIRI. Forty SD rats were randomly divided into four groups $(n=10)$ including Sham operation (Sham), ischemia-reperfusion (IR), sevoflurane preconditioning group (Sevo-Pre) and sevoflurane post-conditioning (Sevo-Post) groups. Perfusion was performed using ex vivo heart perfusion. The baseline values of cardiac function were recorded in each group at the end of balanced perfusion and after $60 \mathrm{~min}$ of reperfusion. Myocardial infarct size (MIS) was calculated at the end of perfusion using TTC staining. Levels of HIF-1 $\alpha$ and caspase-3 protein and HIF-1 $\alpha$ (western blotting) and Bcl-2 mRNA (RT-qPCR) were detected at the end of reperfusion. Our results showed no significant differences in cardiac function between the groups at the end of the balanced perfusion. After reperfusion for $60 \mathrm{~min}$, however, the cardiac functions of the Sevo-Pre and Sevo-Post groups were significantly better than those in the IR group, and the MIS at the end of reperfusion was significantly decreased. Western blotting and RT-qPCR showed that expression of HIF-1 $\alpha$ protein was significantly increased, expression of caspase-3 protein was significantly decreased and expression of HIF-1 $\alpha$ and Bcl-2 mRNA were significantly increased in Sevo-Pre and Sevo-Post groups compared with the levels in the IR group at the end of reperfusion. There were no significant differences in experimental results between Sevo-Pre and Sevo-Post groups. Our data support the idea that sevoflurane can improve MIRI in rats by improving cardiac function and reducing MIS. This protective effect seems to be achieved by activation of HIF-1 $\alpha$ and inhibition of caspase-3.
\end{abstract}

Correspondence to: Dr Mingsheng Zhang, Department of Anesthesiology, Jiangxi Provincial People's Hospital, 92 Aiguo Road, Donghu, Nanchang, Jiangxi 330006, P.R. China

E-mail: pnx7y6@163.com

Key words: sevoflurane, myocardial ischemia-reperfusion injury, HIF-1 $\alpha$, caspase-3

\section{Introduction}

Myocardial ischemia-reperfusion injury (MIRI) refers to the damage to the myocardium caused by both the ischemia and the restoration of blood flow into the previously ischemic tissue. After reperfusion, the myocardial ultrastructure, function, metabolism and electrophysiological characteristics are further damaged, worsening the patients' condition (1). MIRI can cause irreversible damage to myocardial cells and reduce cell activity. Clinical manifestations of MIRI include sudden drop in blood pressure, heart rate disorders and even sudden death (2). The mechanisms of MIRI include an oxygen free radical (ROS) balance disorder, calcium overload, microvascular injury and leukocyte activation. The prevention and treatment of these pathological changes involve anti-oxidative therapy, calcium overload reducing agents and ischemic preconditioning (3). Recent studies have shown that ischemic post-conditioning has a protective effect upon MIR. Due to its benefits, ischemic postconditioning is now widely used in the clinical practice (4).

Studies have shown that inhalation a certain amounts of anesthetics before ischemia or at the beginning of reperfusion can inhibit MIRI, and protect the myocardium (5). Sevoflurane exhibits several advantages such as short anesthesia induction and recovery time, less irritation, a high safety profile and adjustable depths of anesthesia, and is therefore widely used in cardiac surgery. Studies have shown that sevoflurane can reduce myocardial infarct size (MIS) and mortality (6). The mechanism of MIRI inhibition by anesthetics is similar to that of ischemic preconditioning. Both methods protect the myocardium through activation of multiple pathways including NF-кB, BPI3K/Akt, ERK1/2, ROS regulation, mitoKATP channel and mPTP (5-7).

The hypoxia inducible factor $1 \alpha(\mathrm{HIF}-1 \alpha)$ can induce the expression of a series of downstream target genes under hypoxic conditions such as necrosis, ischemia and injury, and these downstream genes, which are involved in angiogenesis, sugar and iron metabolism and cell proliferation, play important roles in apoptosis (8).

In addition, HIF-1 $\alpha$ plays a key regulatory role in the pathogenesis of ischemic heart disease and can inhibit MIS expansion to improve myocardial function (9). Studies have shown that HIF-1 $\alpha$ overexpression can significantly reduce cell apoptosis, and it alters cysteinyl aspartate specific protease-3 (caspase-3) expression, which in turn plays a decisive role in 
the process of cell apoptosis. Therefore, IR injury can induce changes in HIF-1 $\alpha$ and caspase-3 expression, and it has been proposed that the protection effect of sevoflurane on MIRI may be achieved by locally regulating the expression of HIF-1 $\alpha$ and caspase-3 (10). The aim of this study was to investigate the protection effects of sevoflurane on MIRI and its effect on the expression of HIF-1 $\alpha$ and caspase-3 in a rat model, in order to evaluate this hypothesis and provide new insights for the prevention and treatment of MIRI.

\section{Materials and methods}

Experimental animals. Forty SPF grade male SD rats (weighing 250-300 g) were purchased from Shanghai Experimental Animal Center of the Chinese Academy of Science (Shanghai, China). The rats were randomly divided into four groups (each with 10 rats) including a sham operation group (Sham), an ischemia-reperfusion group (IR), a sevoflurane preconditioning group (Sevo-Pre) and a sevoflurane post-conditioning group (Sevo-Post). All animal experiments were carried out strictly in accordance with the guidelines for care and use of laboratory animals. This study was approved by the Animal Ethics Committee of Jiangxi Provincial People's Hospital Animal Center.

Langendorff heart perfusion model preparation. Rats were anesthetized with $50 \mathrm{mg} / \mathrm{kg}$ nembutal sodium (Sigma, St. Louis, MO, USA), and 1000 U/kg heparin (Aladdin, Shanghai, China) was used for anticoagulation. After anesthesia, the rats were fixed in a supine position, the thoracic cavity was opened and the heart was quickly removed and placed in a pre-cooled $\mathrm{K}-\mathrm{H}$ buffer saturated with $95 \% \mathrm{O}_{2}$ and $5 \% \mathrm{CO}_{2}$ mixed gas. Through aorta retrograde intubation, the heart was suspended in the Langendorff perfusion device, and perfusion was performed using $\mathrm{K}-\mathrm{H}$ buffer at $37^{\circ} \mathrm{C}$ with $80 \mathrm{mmHg}$ constant pressure. The left auricle was cut and a latex capsule was inserted into the left atrium. A balloon catheter was connected to a pressure sensor, and the MP1000 physiological signal acquisition system (Biopac Systems, Inc., Goleta, CA, USA) was used to monitor cardiac function parameters including heart rate (HR), left ventricular development pressure (LVDP), left ventricular end diastolic pressure (LVEDP) and the maximum rate of left ventricular $(\mathrm{LV})$ pressure rise $\left( \pm \mathrm{dp} / \mathrm{dt}_{\max }\right)$.

Experimental method for isolated heart perfusion. The hearts in the Sham group were perfused for 150 min. Hearts in the I-R group were subjected to balanced perfusion for $30 \mathrm{~min}$, ischemia for $30 \mathrm{~min}$ and then reperfusion for $90 \mathrm{~min}$. The Sevo-Pre group hearts were treated with balanced perfusion for 15 min, reperfusion with K-H buffer containing $1 \mathrm{MAC}$ sevoflurane (Shanghai Hengrui Pharmaceutical, Shanghai, China) for $10 \mathrm{~min}$, washing out for $5 \mathrm{~min}$, ischemia for $30 \mathrm{~min}$ and finally reperfusion for $90 \mathrm{~min}$. The Sevo-Post group hearts were subjected to balanced perfusion for $30 \mathrm{~min}$, ischemia for 30 min, next reperfusion with K-H buffer containing 1 MAC sevoflurane (Shanghai Hengrui Pharmaceutical) for $10 \mathrm{~min}$, washing out for $5 \mathrm{~min}$ and then reperfusion for $90 \mathrm{~min}$.

MIS determination. After the completion of ischemia and perfusion experiments, the hearts were quickly removed from
Table I. Sequence of primers used in real-time qPCR.

\begin{tabular}{ll}
\hline Primer & \multicolumn{1}{c}{ Sequence (5'-3') } \\
\hline HIF-1 $\alpha$ & F: GACACCGCGGGCACCGATTC \\
& R: TGCTTCGCCGAGATCGTGCTG \\
Bcl-2 & F: TGAACCGGCATCTGCACAC \\
& R: CGTCTTCAGAGACAGCCAGGAG \\
ACTB & F: CAGGGCGTGATGGTGGGCA \\
& R: CAAACATCATCTGGGTCATCTTCTC
\end{tabular}

F, forward; R, reverse.

the $\mathrm{KH}$ solution and stored in a refrigerator at $-20^{\circ} \mathrm{C}$. Each heart was transversally cut along the apex to the bottom, into $5-\mu \mathrm{m}$ slices, the remaining tissue was saved for other experiments (in liquid nitrogen). The slices were then incubated in $0.1 \mathrm{~mol} / 1$ phosphate buffer containing $1 \% \mathrm{TTC}$ at $37^{\circ} \mathrm{C}$ for $20 \mathrm{~min}$ in the dark. With this treatment the living myocardium can be seen to be brick red in color, while the infarcted myocardium appears greyish-white. The excess dye was washed out with ultrapure water and the Image-Pro Plus software (Media Cybernetics, Rockville, MD, USA) was used to calculate the proportion of MIS to total myocardial area.

Western blotting. After reperfusion, heart tissues were removed from the liquid nitrogen storage and homogenized with lysing buffer (Beyotime Biotechnology, Jiangsu, China). The homogenate was then centrifuged at $4^{\circ} \mathrm{C}$ to collect the supernatant containing the soluble fraction. The protein concentration was measured using a Modified BCA kit (Sangon, Shanghai, China). SDS-PAGE was performed using $50 \mu \mathrm{g}$ protein from each sample, followed by transfer to a PVDF membrane. After blocking with blocking solution at room temperature for $2 \mathrm{~h}$, each membrane was incubated with primary rabbit monoclonal HIF-1 $\alpha$ antibody (dilution, 1:500; cat. no. ab51608); rabbit polyclonal caspase-3 antibody (dilution, 1:500; cat. no. ab13847) and rabbit polyclonal $\beta$-actin antibody (dilution, 1:500; cat. no. ab8227) at $4^{\circ} \mathrm{C}$ overnight. Next day, each membrane was washed three times with TBST followed by incubation with secondary goat anti-rabbit (HRP) IgG antibody (dilution, 1:2,000; cat. no. ab6721) at room temperature for $2 \mathrm{~h}$. All antibodies were all purchased from Abcam (Cambridge, MA, USA).

Bands were developed using an ECL detection system (Thermo Fisher Scientific, Waltham, MA, USA), and ImageJ software (National Institutes of Health, Bethesda, MD, USA) was used to calculate the gray value of bands with $\beta$-actin as endogenous control, to obtain relative quantities. All antibodies used were purchased from Cell Signaling Technology, Beverly, MA, USA.

Quantitative real-time polymerase chain reaction ( $q R T-P C R$ ). All reagents used to perform the qRT-PCR (Prime Script ${ }^{\circledR}$ RT Reagent kit with gDNA Eraser and SYBR ${ }^{\circledR}$ Premix Ex $\mathrm{Taq}^{\mathrm{TM}}$ II) were purchased from Takara (Dalian, Liaoning, China) and manufacturer's instructions were followed. Briefly, 
Table II. Effect of sevoflurane treatment on hemodynamic parameters of IR treated hearts.

\begin{tabular}{|c|c|c|c|c|c|}
\hline Group & HR/beats $\cdot \min ^{-1}$ & $\mathrm{LVDP} / \mathrm{kPa}$ & LVEDP/kPa & $+\mathrm{dp} / \mathrm{dt}_{\max } / \mathrm{kPa} \cdot \mathrm{s}^{-1}$ & $-\mathrm{dp} / \mathrm{dt}_{\max } / \mathrm{kPa} \cdot \mathrm{s}^{-1}$ \\
\hline \multicolumn{6}{|c|}{ End of balanced perfusion } \\
\hline Sham & $307 \pm 13$ & $15.7 \pm 1.4$ & $0.74 \pm 0.13$ & $436 \pm 17$ & $338 \pm 24$ \\
\hline I-R & $310 \pm 11$ & $15.9 \pm 2.0$ & $0.79 \pm 0.24$ & $452 \pm 13$ & $324 \pm 31$ \\
\hline Sevo-Pre & $303 \pm 18$ & $16.3 \pm 1.8$ & $0.82 \pm 0.11$ & $446 \pm 21$ & $340 \pm 17$ \\
\hline Sevo-Post & $310 \pm 12$ & $15.3 \pm 2.2$ & $0.77 \pm 0.16$ & $449 \pm 23$ & $334 \pm 24$ \\
\hline \multicolumn{6}{|c|}{60 min reperfusion } \\
\hline Sham & $298 \pm 12$ & $16.3 \pm 1.5$ & $0.78 \pm 0.13$ & $424 \pm 16$ & $328 \pm 18$ \\
\hline I-R & $173 \pm 15^{\mathrm{a}}$ & $7.8 \pm 2.6^{\mathrm{a}}$ & $4.34 \pm 1.35^{\mathrm{a}}$ & $283 \pm 12^{\mathrm{a}}$ & $189 \pm 23^{\mathrm{a}}$ \\
\hline Sevo-Pre & $256 \pm 13^{c}$ & $10.2 \pm 1.5^{\mathrm{b}}$ & $3.26 \pm 0.45^{\mathrm{b}}$ & $326 \pm 15^{\mathrm{b}}$ & $223 \pm 13^{b}$ \\
\hline Sevo-Post & $263 \pm 17^{\mathrm{c}}$ & $11.0 \pm 1.4^{\mathrm{b}}$ & $3.49 \pm 0.56^{\mathrm{b}}$ & $324 \pm 21^{\mathrm{b}}$ & $234 \pm 17^{b}$ \\
\hline
\end{tabular}

Compared with Sham group, ${ }^{\mathrm{a}} \mathrm{P}<0.01$; compared with I-R group, ${ }^{\text {b}} \mathrm{P}<0.05$; compared with I-R group, ${ }^{\mathrm{c}} \mathrm{P}<0.01$.

total RNA was extracted from the left ventricular myocardium after perfusion (TRIzol reagent). cDNA was synthesized by reverse transcription using $200 \mathrm{ng}$ total RNA, and the cDNA was used to prepare the real-time PCR reaction system. PCR reaction was performed using CFX-96 Real-Time PCR Detection System (Bio-Rad, New York, NY, USA). Data were processed using the $2^{-\Delta \Delta c t}$ method using data from the Sham group as control group and data from the $\beta$-actin gene to normalize the data. All primers were synthesized by Sangon. Primer sequences are listed in Table I.

Statistical analysis. The statistical analysis was performed using the SPSS 17.0 software (SPSS, IL, USA). All experiments were repeated three times and data were expressed as mean \pm SD. Single factor analysis of variance and two-tailed t-tests were performed for comparisons between groups. $\mathrm{P}<0.05$ was considered to indicate a statistically significant difference.

\section{Results}

Effect of sevoflurane treatment on cardiac function. Through the real-time monitoring of cardiac function parameters, data were collected at the end of the balanced perfusion and after 60 min of reperfusion. As shown in Table II, no significant differences were found in cardiac function at the end of the balanced perfusion between the groups $(\mathrm{P}>0.05)$. However, after $60 \mathrm{~min}$ of reperfusion, the cardiac function was significantly reduced in the I-R group compared with that in the Sham group $(\mathrm{P}<0.05)$, indicating that MIRI can significantly reduce cardiac function. Compared with I-R group, the values of HR, LVDP and $\pm d p / \mathrm{dt}_{\max }$ were significantly increased but the value of LVEDP was significantly decreased in the Sevo-Pre and Sevo-Post groups, suggesting that sevoflurane (1 MAC) treatment can significantly enhance systolic and diastolic function of the myocardium after reperfusion in rats. There were no significant differences between Sevo-Pre and Sevo-Post group.

Effect of sevoflurane treatment on MIS. After reperfusion, the MIS of each group was determined by TTC staining. As shown in Fig. 1, compared with the Sham group, MIS

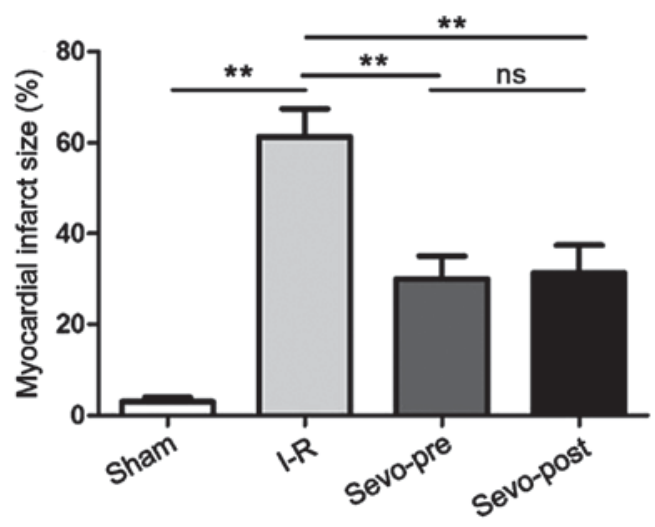

Figure 1. Graph showing effect of sevoflurane treatment on MIS, as evidenced on TTC staining. ${ }^{* *} \mathrm{P}<0.01,{ }^{\text {ns}} \mathrm{P}>0.05$.

was significantly increased in the I-R group. Compared with the I-R group, MIS was significantly decreased in the Sevo-Pre and Sevo-Post groups, indicating that sevoflurane treatment can significantly reduce MIS in rats after MIRI. There were no significant differences between Sevo-Pre and Sevo-Post groups $(\mathrm{P}>0.05)$.

Effect of sevoflurane treatment on the expression of HIF-1 $\alpha$ and caspase-3 protein. Western blotting was used to perform a semi-quantitative analysis of the levels of HIF- $1 \alpha$ and caspase-3 protein in cardiomyocytes after reperfusion. As shown in Fig. 2, compared with the Sham group, the expression levels of HIF-1 $\alpha$ were decreased and the expression levels of caspase-3 were increased in the I-R group. In addition, compared with the I-R group, HIF-1 $\alpha$ was significantly increased and caspase-3 was significantly decreased in Sevo-Pre and Sevo-Post groups. No significant differences were found between Sevo-Pre and Sevo-Post groups. The results suggest that sevoflurane treatment can significantly increase the expression of HIF-1 $\alpha$ protein and decrease the expression of caspase- 3 protein $(\mathrm{P}>0.05)$.

Effect of sevoflurane treatment on the expression of HIF-1 $\alpha$ and $\mathrm{Bcl}$-2. qRT-PCR was used to perform a semi-quantitative 
A
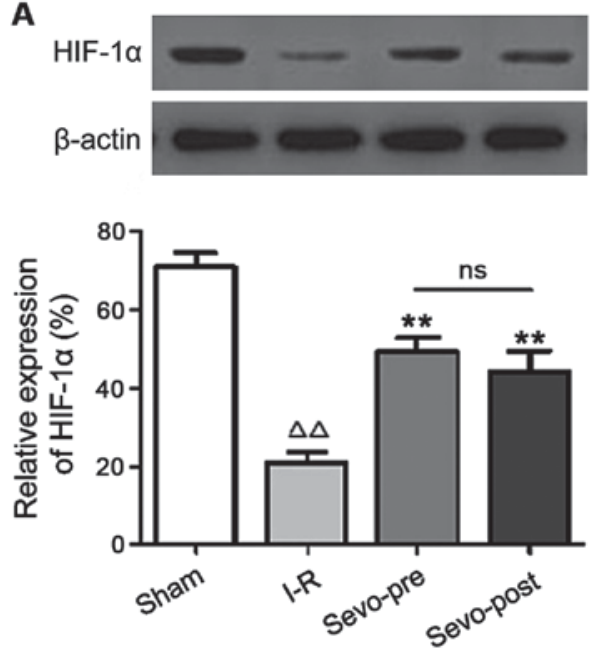

B
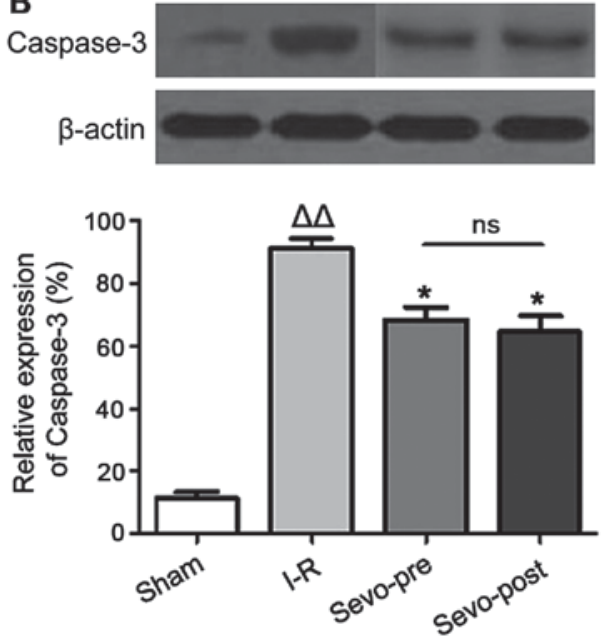

Figure 2. Effect of sevoflurane treatment on the expression of (A) HIF-1 $\alpha$ and (B) caspase-3 proteins as seen by western blot analysis. Compared with Sham group, ${ }^{\triangle} \triangle \mathrm{P}<0.01$; compared with I-R group, ${ }^{*} \mathrm{P}<0.05$; compared with I-R group, ${ }^{* *} \mathrm{P}<0.01$; ${ }^{\text {ns }} \mathrm{P}>0.05$. $\beta$-actin was used to normalize the relative expression levels across groups.

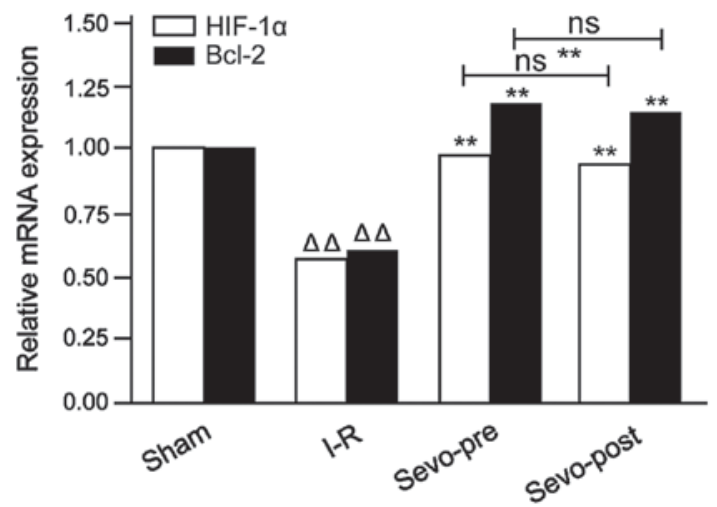

Figure 3. Graph showing effect of sevoflurane treatment on the relative expression of HIF-1 $\alpha$ and Bcl-2 mRNAs. Compared with Sham group, ${ }^{\triangle} \triangle \mathrm{P}<0.01$; compared with I-R group, ${ }^{* *} \mathrm{P}<0.01$; ${ }^{\text {ns }} \mathrm{P}>0.05$. $\beta$-actin was used to normalize expression levels for comparisons.

analysis of the expression levels of HIF-1 $\alpha$ and Bcl-2 mRNA in cardiomyocytes after reperfusion. As shown in Fig. 3, compared with the Sham group, the expression levels of HIF-1 $\alpha$ and $\mathrm{Bcl}-2$ mRNA were significantly reduced in the I-R group. Moreover, compared with I-R group, the expression levels of HIF- $1 \alpha$ and Bcl-2 mRNA were significantly increased in the Sevo-Pre and Sevo-Post groups. No significant differences were found between Sevo-Pre and Sevo-Post groups. The data indicate that sevoflurane treatment can significantly increase the expression levels of HIF-1 $\alpha$ and Bcl-2 mRNA.

\section{Discussion}

Inhalation of a certain dose of sevoflurane before myocardial ischemia or during the early stages of reperfusion has been shown to be able to protect the myocardium by inhibiting myocardial abnormalities during reperfusion through the activation of specific signaling pathways, and this myocardial protection is similar to that of ischemic preconditioning (11). Studies have shown that sevoflurane pre-conditioning and post-conditioning can enhance myocardial contractility and diastolic ability, improve cardiac function, and reduce myocardial infarction caused by IR, cardiovascular disorders and other symptoms (12). In order to confirm the cardioprotective effect of sevoflurane during MIRI, we carried out MIRI in vitro simulations using rat hearts for the model. Through real-time monitoring of cardiac function parameters, we found that sevoflurane pre-/post-conditioning can significantly improve left ventricular $\mathrm{HR}, \mathrm{LVDP} \pm \mathrm{dp} / \mathrm{dt}_{\max }$, and reduce left ventricular LVEDP in rat hearts during the late stages of reperfusion, indicating that sevoflurane treatment can enhance myocardial contractility and diastolic ability of IR hearts. In addition, sevoflurane treatment also reduced MIS after reperfusion compared with the control group, indicating that sevoflurane can protect the myocardium against MIRI.

Apoptosis, which is regulated by anti-apoptotic factor Bcl-2 and apoptotic hydrolase caspase-3 through a series of complex processes, is an important molecular mechanism in the process of MIRI cell death and plays an important role in IR injury and prognosis $(13,14)$. Studies have shown that sevoflurane can activate the $\mathrm{NF}-\kappa \mathrm{B}$ pathway, increase $\mathrm{Bcl}-2$ expression and reduce expression levels of intracellular adhesion factor-1 (CAM-1) and tumor necrosis factor- $\alpha$ (TNF- $\alpha$ ), so as to reduce the expression levels of caspase- 3 protein, thus inhibiting apoptosis (15). In addition, sevoflurane treatment can reduce expression levels of Beclin-1 and LC-I/II, relieving IR damage by inhibiting excessive autophagy $(16,17)$. HIF-1 $\alpha$ has been shown to play an important role in the reduction of MIS and the decrease of apoptosis rates after ischemic preconditioning. HIF-1 $\alpha$ can attenuate myocardial IR injury by enhancing the activity of heme oxygenase-1 (18).

In order to investigate the effects of sevoflurane on HIF-1 $\alpha$ expression and cell apoptosis, expression levels of HIF-1 $\alpha$, Bcl-2 and caspase-3 were quantitatively analyzed. Our results comparing with those in the control group, showed that sevoflurane treatment can significantly increase the expression of HIF- $1 \alpha$ mRNA and protein and Bcl- 2 mRNA. Moreover, it can decrease the expression level of caspase-3 protein. Taken together, these facts indicate that HIF-1 $\alpha$ can regulate the 
apoptosis of cardiomyocytes by regulating the expression of caspase-3 through a yet-undisclosed mechanism. It is known that activation of HIF-1 $\alpha$ can induce the expression of downstream genes involved in cytoprotection, so as to inhibit cell apoptosis and reduce myocardial injury caused by IR $(19,20)$.

In conclusion, our study confirmed that sevoflurane (1MAC) treatment can improve the function parameters of hearts undergoing IR and reduces MIS. The protection is probably due to sevoflurane activating the expression of HIF-1 $\alpha$ and inhibiting the expression of caspase-3. Our findings provide new insights for the diagnosis and treatment of MIRI.

\section{References}

1. Goldhaber JI and Weiss JN: Oxygen free radicals and cardiac reperfusion abnormalities. Hypertension 20: 118-127, 1992.

2. Hu Q, Chen J, Jiang C and Liu HF: Effect of peroxisome proliferator-activated receptor gamma agonist on heart of rabbits with acute myocardial ischemia/reperfusion injury. Asian Pac J Trop Med 7: 271-275, 2014.

3. Bolli R: The early and late phases of preconditioning against myocardial stunning and the essential role of oxyradicals in the late phase: An overview. Basic Res Cardiol 91: 57-63, 1996.

4. Xia A, Xue Z, Wang W, Zhang T, Wei T, Sha X, Ding Y and Zhou W: Naloxone postconditioning alleviates rat myocardial ischemia reperfusion injury by inhibiting JNK activity. Korean J Physiol Pharmacol 18: 67-72, 2014.

5. Yao YY, Zhu MH, Zhang FJ, Wen CY, Ma LL, Wang WN Wang CC, Liu XB, Yu LN, Qian LB, et al: Activation of Akt and cardioprotection against reperfusion injury are maximal with only five minutes of sevoflurane postconditioning in isolated rat hearts. J Zhejiang Univ Sci B 14: 511-517, 2013.

6. Ceyhan D, Tanrıverdi B and Bilir A: Comparison of the effects of sevoflurane and isoflurane on myocardial protection in coronary bypass surgery. Anadolu Kardiyol Derg 11: 257-262, 2011.

7. Gong JS, Yao YT, Fang NX and Li LH: Sevoflurane postconditioning attenuates reperfusion-induced ventricular arrhythmias in isolated rat hearts exposed to ischemia/reperfusion injury. Mol Biol Rep 39: 6417-6425, 2012.

8. Fandrey J, Gorr TA and Gassmann M: Regulating cellular oxygen sensing by hydroxylation. Cardiovasc Res 71: 642-651, 2006.

9. Kido M, Du L, Sullivan CC, Li X, Deutsch R, Jamieson SW and Thistlethwaite PA: Hypoxia-inducible factor 1-alpha reduces infarction and attenuates progression of cardiac dysfunction after myocardial infarction in the mouse. J Am Coll Cardiol 46: 2116-2124, 2005.
10. Peng XH, Karna P, Cao Z, Jiang BH, Zhou M and Yang L: Cross-talk between epidermal growth factor receptor and hypoxia-inducible factor-1alpha signal pathways increases resistance to apoptosis by up-regulating survivin gene expression. J Biol Chem 281: 25903-25914, 2006.

11. Kodaka M, Johansen JW and Sebel PS: The influence of gender on loss of consciousness with sevoflurane or propofol. Anesth Analg 101: 377-381, 2005.

12. Kehl F, Krolikowski JG, Tessmer JP, Pagel PS, Warltier DC and Kersten JR: Increases in coronary collateral blood flow produced by sevoflurane are mediated by calcium-activated potassium (BKCa) channels in vivo. Anesthesiology 97: 725-731, 2002.

13. Meng XY, Yu HL, Zhang WC, Wang TH, Mai X, Liu HT and Xu RC: ZFP580, a novel zinc-finger transcription factor, is involved in cardioprotection of intermittent high-altitude hypoxia against myocardial ischemia-reperfusion injury. PLoS One 9: e94635, 2014.

14. Eefting F, Rensing B, Wigman J, Pannekoek WJ, Liu WM, Cramer MJ, Lips DJ and Doevendans PA: Role of apoptosis in reperfusion injury. Cardiovasc Res 61: 414-426, 2004.

15. Hu G, Salem MR and Crystal GJ: Role of adenosine receptors in volatile anesthetic preconditioning against neutrophil-induced contractile dysfunction in isolated rat hearts. Anesthesiology 103: 287-295, 2005.

16. Han Z, Cao J, Song D, Tian L, Chen K, Wang Y, Gao L, Yin Z, Fan Y and Wang C: Autophagy is involved in the cardioprotection effect of remote limb ischemic postconditioning on myocardial ischemia/reperfusion injury in normal mice, but not diabetic mice. PLoS One 9: e86838, 2014.

17. Cao X, Chen A, Yang P, Song X, Liu Y, Li Z, Wang X, Wang L and $\mathrm{Li} \mathrm{Y}$ : Alpha-lipoic acid protects cardiomyocytes against hypoxia/reoxygenation injury by inhibiting autophagy. Biochem Biophys Res Commun 441: 935-940, 2013.

18. Cai Z, Zhong H, Bosch-Marce M, Fox-Talbot K, Wang L, Wei C, Trush MA and Semenza GL: Complete loss of ischaemic preconditioning-induced cardioprotection in mice with partial deficiency of HIF-1 alpha. Cardiovasc Res 77: 463-470, 2008.

19. Liu LX, Lu H, Luo Y, Date T, Belanger AJ, Vincent KA, Akita GY, Goldberg M, Cheng SH, Gregory RJ, et al: Stabilization of vascular endothelial growth factor mRNA by hypoxia-inducible factor 1. Biochem Biophys Res Commun 291: 908-914, 2002.

20. Mazure NM, Brahimi-Horn MC, Berta MA, Benizri E, Bilton RL, Dayan F, Ginouvès A, Berra E and Pouysségur J: HIF-1: Master and commander of the hypoxic world. A pharmacological approach to its regulation by siRNAs. Biochem Pharmacol 68: 971-980, 2004. 